\title{
混合式教学模式在结构化学课程中的探索与实践
}

关丽丽 ${ }^{*}$, 蔡颖, 宋金玲, 肖淑艳, 胡锋, 赵金金

内蒙古科技大学材料与冶金学院, 内蒙古 包头 014010

摘要: 针对结构化学的特点, 开展混合式教学模式的探索与实践。将网络教学平台的自主学习与传统面授进行优势 互补, 对抽象化知识点进行可视化表达, 调动学生学习的积极性和主动性, 并根据学习效果及时调整线下授课方 案。混合式教学模式的应用, 充分体现了教师的主导作用及学生的主体作用, 取得了较好的教学效果。

关键词: 结构化学; 混合式教学; 教学效果

中图分类号: G64; O6

\section{Exploration and Practice of Blended Teaching Mode in the Structural Chemistry Course}

\author{
Lili Guan *, Ying Cai, Jinling Song, Shuyan Xiao, Feng Hu, Xin Zhao \\ School of Materials and Metallurgy, Inner Mongolia University of Science and Technology, Baotou 014010, \\ Inner Mongolia Autonomous Region, China.
}

\begin{abstract}
In view of the characteristics of structural chemistry, the exploration and practice of mixed teaching mode were carried out. The network teaching platform's independent study and the traditional face-to-face instruction superiority are carried out to complement each other. The visual expression of abstract knowledge points arouses the enthusiasm and initiative of students. The offline teaching plan is adjusted in time according to the learning effect. The application of hybrid teaching mode fully illustrates the leading role of teachers and the main role of students, and achieved better teaching results.
\end{abstract}

Key Words: Structural chemistry; Blended teaching; Teaching effect

结构化学是利用量子力学基础, 从微观层次上研究原子、分子、晶体结构及结构与性能关系的 一门基础科学。由于课程的研究对象是抽象的微观体系, 因此理论性较强, 内容晦澀难懂。在课程 学习过程中, 不仅要求学生具有一定的数理知识, 同时要具有严密的逻辑思维能力和一定的空间想 象力, 导致多数学生存在畏难和抵触心理 ${ }^{[1-3]}$ 。此外, 现阶段, 在有限的课堂学时内, 传统的面授、 灌输式的教学方法已无法充分调动学生学习的积极性和主动性, 因此必须针对结构化学课程进行适 当的教学改革, 例如开展模型实习、讨论课、翻转课堂及在线教学等 ${ }^{[4-6]}$ 。本文介绍了线上线下混合 式教学模式在结构化学课程中的探索与实践。

结构化学混合式课程设计与建设方案如图1所示。具体内容包含课前学生的自主学习, 主要为教 师发布的教学平台内容及知识点自测; 线下内容主要针对课程内容中知识点的串讲, 以及针对线上 自主学习中普遍出现的问题进行集中解析, 课后学生对重要知识点进行巩固练习。

收稿: 2021-05-12; 录用: 2021-06-15; 网络发表: 2021-07-01

“通讯作者, Email: guanlili907007@163.com

基金资助：内蒙古科技大学教育教学改革项目(JY2019001) 


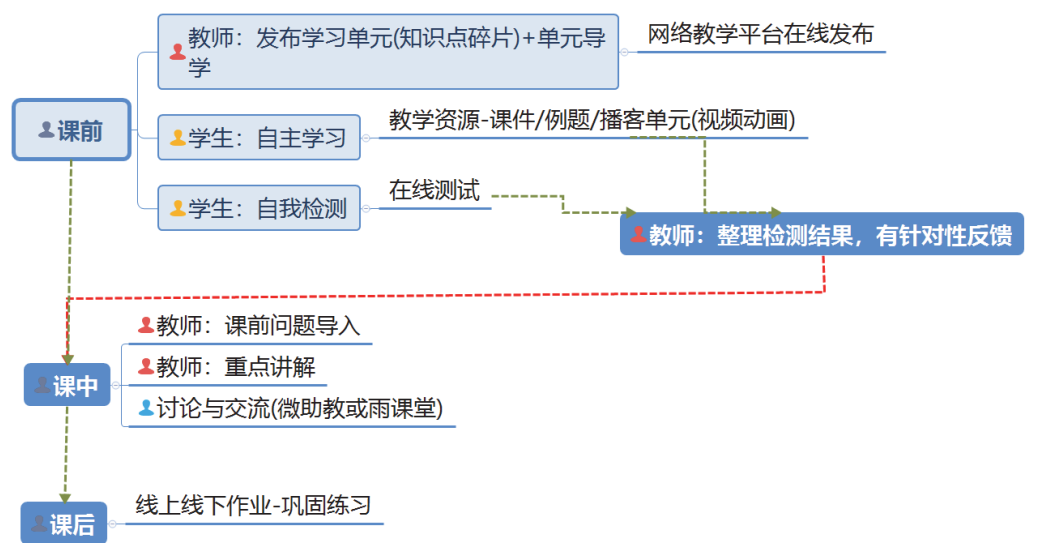

图1 线上、线下混合教学模式方案

\section{1 线上课程平台建设}

本课程基于优慕课在线教育平台开展线上内容建设 ${ }^{[7]}$ 。按照结构化学课程的教学大纲, 将教学 内容划分为学习单元, 各单元学时的分配可查阅平台中 “课程信息” 模块下的教学大纲。每个学习 单元下设置具体的学习内容, 包括课件资源、 “单元导学” 模块及知识点学习。通过思维导图建立 知识点关联性, 帮助学生了解本单元的知识点、学习目标及学习进度。

图2列举了单元4 (分子的对称性)的导学内容。导学中首先明确了教学要求, 并建立单元知识点 导图, 模块中不同颜色代表了知识点的掌握程度, 同时细化每部分的学习进度。例如, 第一个模块 为对称操作和对称元素, 其中有三个重要知识点, 包括旋转轴、对称中心以及镜面。学生了解知识 点掌握程度的同时, 可以按照课前及课后自主安排学习时间, 便于学生从整体上把控学习强度并合 理安排学习进度。

教学要求: 掌握对称元素和对称操作的基本概念 (恒等, 旋转, 反映, 反演等) ; 理解对称操作的 组合规则和对易规则, 理解群的基本概念、分子对称性和分子物理性质之间的关系 (偶极距, 旋光 性）。掌握分子点群的确定步骤; 了解群的表示。

重难点: 对称元素的组合; 分子所属点群的判别。

单元考评: 自主学习20分(在线时长 $120 \mathrm{~min}$ )+单元测试 20 分+作业 10 分, 满分 50 分。
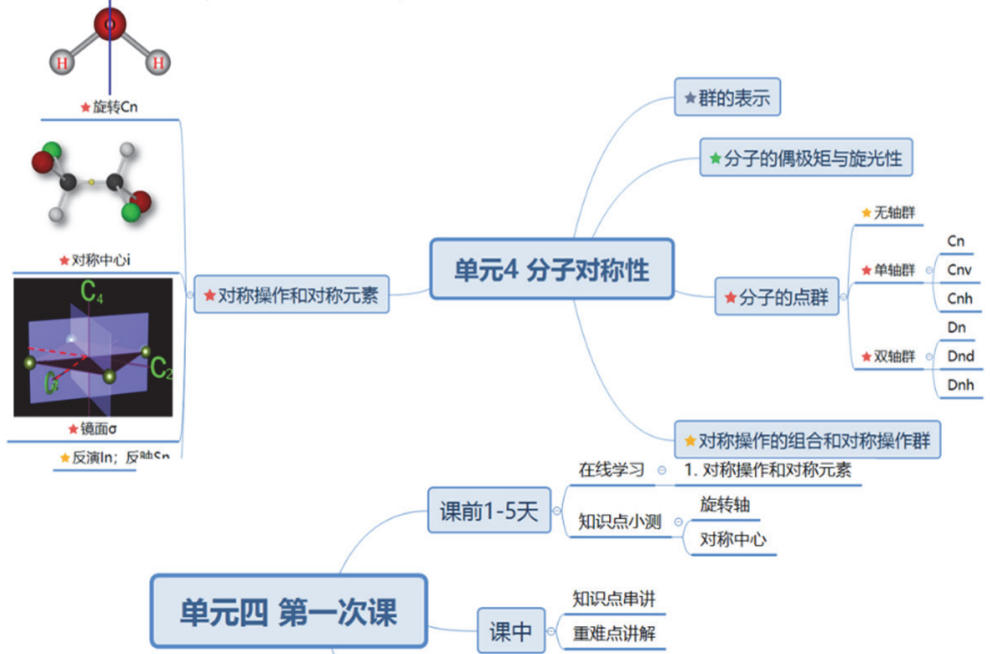

课后1-5天 学习效果反馈-线上线下作业

图2 单元导学(单元 4 “分子的对称性”)

电子版为彩图 
学生通过 “学习单元” 的学习掌握知识点, 并且针对一些需要重点掌握的教学内容, 还可通过 “例题精讲” 模块加深理解。例如在单元4中, 分子全部对称元素及所属点群的判定是这一章节的重 要知识点, 部分学生在单元学习后仍无法准确地进行判断, 经常出现点群判断错误或无法找出全部 的对称元素, 故而在单元4模块下增加了典型分子如苯、间苯三酚、乙烯、环丙烷等的三维立体构型 及动态旋转图, 如图3所示, 并对其进行说明, 使学生通过分子结构可视化深刻理解这一知识点的判 定。此外, 学生也能够通过 “单元小测” 模块及时掌握自主学习的效果。同时, 线上课程平台开设 讨论区, 学生可以通过讨论区与教师及其他同学展开交流讨论, 对不理解的知识点进行沟通, 促进 学习效果的提升。

\section{结构化学 首页 | 基本信息 | 学习刍元 | 知识拓展-晶体结构... $\mid$ 调查间卷 | 课珵活动}

间苯三酚全部对称元素及所属点群

对称元素: $\mathrm{C}_{3}, \sigma_{\mathrm{h}}, \mathrm{E}$ 所属点群: $\mathrm{C}_{3 \mathrm{~h}}$
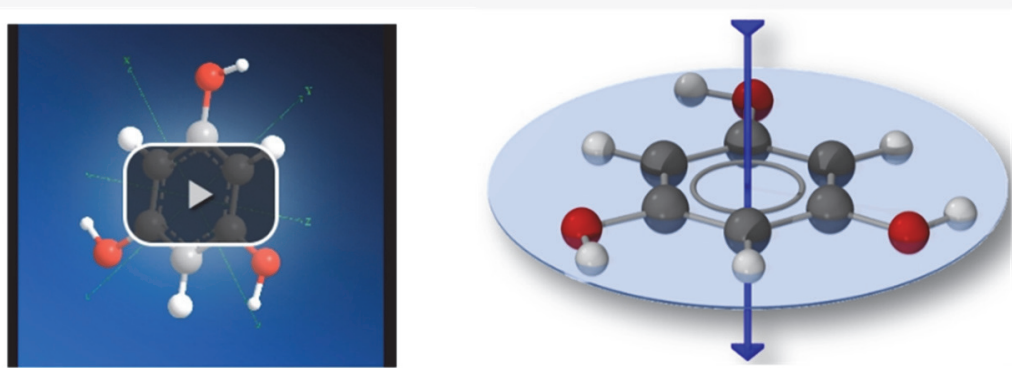

图3 单元4 “例题精讲” 模块下的课程建设内容

此外, 平台增加了 “知识拓展” 模块, 在该模块下学生可自行下载典型晶体如 $\mathrm{CaF}_{2} 、 \mathrm{NaCl} 、$ 金 刚石、石墨等材料的.vesta文件。除了在课堂上搭建晶体球棍模型之外, 还可以通过Vesta晶体结构可 视化软件了解晶系、点阵及晶面指数等内容, 增加学习趣味性 ${ }^{[8]}$ 。例如, 图 4 是学生通过Vesta软件打 开的 $\mathrm{CaF}_{2}$ 和 $\mathrm{NaCl}$ 的晶体结构图。其中, 图 $4 \mathrm{a}$ 中黄色区域表示的是 $\mathrm{CaF}_{2}$ 的 (110)晶面, 由 Vesta工具栏中 的Lattice planes选项实现, 图4b为面心立方 $\mathrm{NaCl}$ 的多面体形式, 从图中可以很清晰地看出 $\mathrm{Cl}^{-}$的配位 数为6, 即八面体配位。通过三维结构的可视化, 在激发学生学习主动性的同时, 能够使学生更好地 掌握晶系、空间点阵等晶体结构的知识点。

(a)

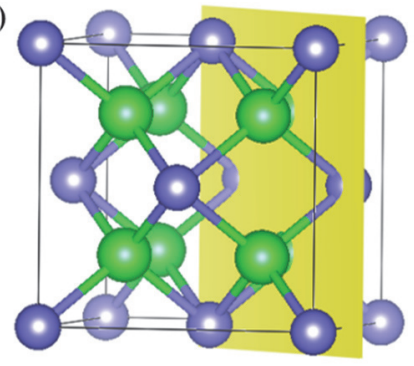

(b)

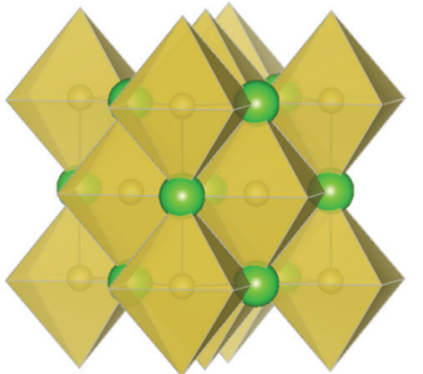

图4 晶体结构图

(a) $\mathrm{CaF}_{2} ;$ (b) $\mathrm{NaCl}$

电子版为彩图

\section{2 线上内容与线下的有机结合}

结构化学课程线下内容的建设, 主要针对线上学习效果进行知识点串讲及重难点解析, 并根据 线上测试的反馈结果, 及时调整线下面授的教学方案。例如, 图5为线上平台单元2 (原子的结构和性 
质)中五个知识点的掌握情况, 汇总样品数为 64 。从分析结果可以看出, 在 “原子的结构和性质” 这 一单元中, 学生对知识点的掌握总体情况良好, $10 \%$ 左右的学生对径向分布函数及多电子原子轨道 能的学习效果不理想。因此, 根据这一反馈结果在线下的面授课程中加以强调, 通过线上反馈结果 及时调整线下课程的内容, 实现线上线下的优势互补。

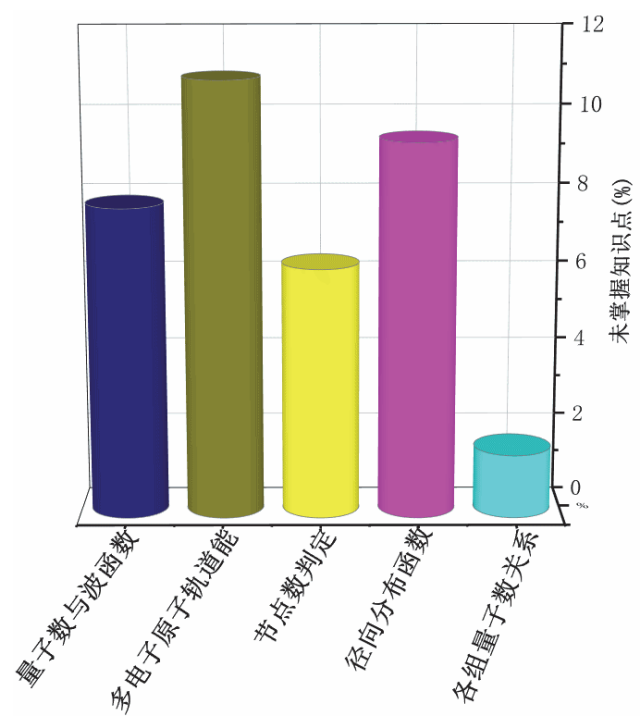

图5 线上平台单元 2 (原子的结构和性质)知识点小测分析结果

同时, 为了调动学生在线下面授课堂中的积极性, 课程采用了微助教平台进行随堂学习检测 $[9]$ 。 例如, 图6所示为线下授课中通过价层电子对互斥理论(VSEPR)判断分子构型这一知识点的考查情 况, 测试以三道选择题的形式出现, 学生参与率为 $80 \%$ 以上, 因此基本上能够调动大多数同学参与 课堂活动。此外, 通过测试发现, 该知识点的正确率在第一次练习时仅为 $50 \%$, 通过教师根据反馈 结果对知识点进行重点解析后, 正确率提高到了 $85 \%$ 、93\%, 因此该方式有助于教师及时了解学生对 知识点的掌握情况, 以便做出调整。

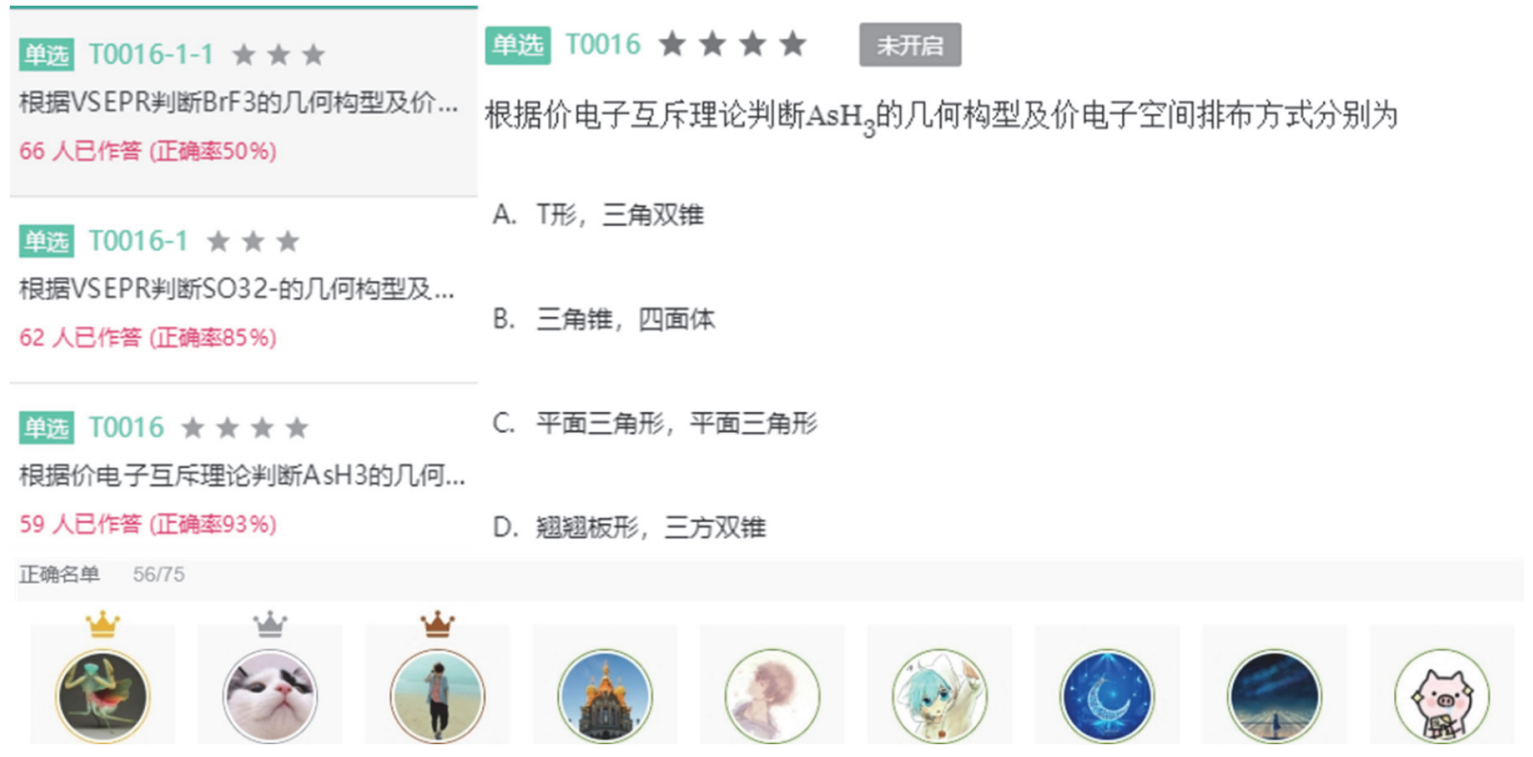

图6 线下授课中微助教平台知识点测试结果图 


\section{3 结语}

将混合式教学模式应用于结构化学课程的教学中, 将线上自主学习与线下面授有机结合起来, 充分利用现代化学习平台及专业软件, 加强学生对抽象知识的理解, 引导学生自主学习, 构建知识 框架, 提高课堂教学效率, 确保课程教学质量。通过线上教学平台, 教师可及时掌握学生学习效果 并根据反馈结果调整线下面授内容, 充分体现了教师的主导作用及学生的主体作用, 使学生取得了 较好的学习效果。

\section{参 考 文 献}

[1] 周公度, 段连运. 结构化学基础. 第5版. 北京: 北京大学出版社, 2017.

[2] 孙宏伟, 陈兰. 大学化学, 2021, 36 (1), 2008056.

[3] 雷鸣, 杨作银, 李亚平, 蒲敏. 大学化学, 2020, 35 (5), 98.

[4] 毛双. 大学化学, 2020,35 (5), 19.

[5] 饶立, 任彦亮, 万坚, 李永健. 大学化学, 2018, 33 (11), 34.

[6] 孙宏伟, 陈兰. 大学化学, 2018, 33 (6), 18.

[7] 优慕课官网. [2021-05-02]. http://opencourse.umooc.com.cn/index.do

[8] Momma, K.; Izumi, F. J. Appl. Crystallogr. 2011, 44, 1272.

[9] 微助教官网. [2021-05-02]. https://portal.teachermate.com.cn/ 